\title{
ALGUNAS OBSERVACIONES ACERCA DEL SIGNIFICADO LÓGICO DE LAS CATEGORÍAS PURAS
}

\author{
Martín Arias Albisu*
}

\begin{abstract}
Resumen
El objetivo de nuestro artículo es efectuar algunas precisiones acerca del concepto de significado lógico de las categorías puras empleado por Kant en su Crítica de la razón pura. En algunos pasajes, Kant afirma que las categorías puras, a saber, consideradas con independencia de los esquemas trascendentales, conservan un significado puramente lógico. En otros pasajes, Kant da a entender que las categorías así consideradas carecen de todo tipo de significado. En primer lugar, mostraremos que, pese a esta vacilación en el texto kantiano, la doctrina del significado lógico de las categorías puras es parte integrante de la filosofía crítica. En segundo lugar, determinaremos qué es el significado lógico de las categorías puras. Afirmaremos que las categorías pueden referirse a intuiciones espacio-temporales de nuestra sensibilidad tan sólo gracias a los esquemas trascendentales. Sostendremos que, dado que en las categorías puras se hace abstracción de esta referencia a intuiciones determinadas, el significado lógico de esas categorías es enteramente indeterminado. El significado lógico de una categoría pura es el pensamiento de la referencia determinante de lo pensado en esa categoría a las indeterminadas intuiciones en general.
\end{abstract}

Palabras clave: Kant, Categoría pura, Esquema trascendental, Significado lógico, Significado empírico

\begin{abstract}
The aim of this paper is to clarify the concept of logical meaning of pure categories as used by Kant in his Critique of Pure Reason. In some passages, Kant states that pure categories, that is, categories considered independently of transcendental schemata, keep an entirely logical meaning. In some other passages, Kant indicates that the categories so considered lack any kind of meaning. In the
\end{abstract}

* Doctor en Humanidades y Artes con Mención en Filosofía por la UNR. Se desempeña como Becario Postdoctoral de CONICET. Por otro lado, participa de Proyectos de Investigación de la UNR, CONICET y FONCyT. Dirección electrónica: arias.martin@gmail.com 
first place, we show that, despite this hesitation in the Kantian text, the doctrine of the logical meaning of pure categories is an integral part of critical philosophy. In the second place, we examine what is the logical meaning of pure categorics. We argue that categories can refer to spatio-temporal intuitions of our sensibility only thanks to transcendental schemata. We hold that, since in pure categorics abstraction is made of this reference to determinate intuitions, the logical meaning of these categories is entirely undetermined. The logical meaning of a pure category is the thought of the determining reference of what is thought in that category to indeterminate intuitions in general.

Keywords: Kant, Pure Category, Transcendental Schema, Logical Meaning, Empirical Meaning

\section{I.}

El capítulo "Del esquematismo de los conceptos puros del entendimiento" de la Crítica de la razón pura de Kant procura mostrar cómo, o bajo qué condiciones, es posible la aplicación de las categorías a la multiplicidad empírica. Según Kant, esta aplicación sólo puede tener lugar por intermedio de los esquemas trascendentales. Los esquemas trascendentales posibilitan la aplicación de las categorías a la multiplicidad empírica. Mediante tal aplicación, la multiplicidad empírica se constituye como objeto empírico. Los esquemas trascendentales, entonces, hacen posible que haya objetos empíricos de una experiencia humana única correspondientes a las categorías. En virtud de esta circunstancia, las categorías adquieren significado objetivo o empírico tan sólo gracias a los esquemas trascendentales.

En el capítulo del esquematismo Kant afirma que las categorías puras, a saber, consideradas haciendo abstracción de sus esquemas, conservan un significado, aunque meramente lógico (cfr. 146-147/B 186) ${ }^{1}$. Esta

${ }^{1}$ Hacemos referencia a la Kritik der reinen Vernunft (Crítica de la razón pura), tal como se acostumbra, de acuerdo con la paginación de la edición original. Con " $\mathrm{A}$ " mentamos la primera edición, de 1781, mientras que con " $\mathrm{B}$ " designamos la segunda, de 1787. Todas las citas de la obra mencionada están tomadas de la traducción española de Mario Caimi (Crítica de la razón pura, Buenos Aires, Colihue, 2007). Tanto en el caso de $K r V$ como en el de otras obras kantianas, hemos empleado los textos incluidos en la edición académica de los trabajos del filósofo de 
afirmación presenta dos dificultades. En primer lugar, entra en contradicción con otros pasajes de la Crítica de la razón pura en los que se afirma que las categorías puras carecen de significado (cfr., por ejemplo, B 148-149). En segundo lugar, no queda claro en qué consiste el significado lógico de las categorías puras.

Nuestro trabajo se propone abordar los problemas mencionados. En primer lugar, intentaremos mostrar que, pese a la vacilación que presenta el texto kantiano en este respecto, la doctrina del significado lógico de las categorías puras es parte integrante de la filosofía crítica. En segundo lugar, procuraremos determinar en qué consiste exactamente el significado lógico mencionado. Con este fin, presentaremos una reconstrucción del significado lógico de la categoría pura de causalidad y dependencia.

Cabe destacar que el análisis del significado lógico de las categorías puras presupone un examen previo de la concepción kantiana del significado. Por este motivo procuraremos exponer, a partir de las indicaciones dispersas efectuadas por Kant, su teoría del significado. Para esta tarea nos serviremos críticamente de los resultados de dos trabajos que intentaron reconstruir la teoría kantiana de la referencia, el sentido y el significado ${ }^{2}$. Presentaremos únicamente las tesis centrales que consideremos sustentadas por el texto kantiano. El análisis de estas tesis nos permitirá arribar posteriormente a un conocimiento adecuado de la distinción entre significado empírico y significado lógico de las categorías ${ }^{3}$.

J. P. Nolan ha planteado el problema de si Kant distingue entre "Sinn" (sentido) y "Bedeutung" (significado). Nolan señala que Kant nunca estableció una distinción sistemática entre los dos términos. En efecto, en muchos pasajes de $\mathrm{KrV}$ estos términos son usados conjuntamente ${ }^{4}$, lo cual sugiere que se los considera sinónimos. Además, en el siguiente pasaje se los

Königsberg (Kant's gesammelte Scbriften, Berlin, Königlich Preußischen Akademie der Wissenschaften, 1902 ss.). Mentamos esta edición de conjunto con la sigla "AA" (Akademie-Ausgabe) y damos a continuación el número de tomo (en romanos) y de página (en arábigos).

${ }^{2}$ Nos referimos a Nolan, J. P., "Kant on meaning: Two Studies", Kant Studien, 70, 2, 1979; y Loparic, Z., A semântica transcendental de Kant, Campinas, UNICAMP, 2000.

${ }^{3}$ Cfr. II.

${ }^{4}$ Cfr. B 149, A 155/B 194, A 156/B 195. 
usa explícitamente de manera intercambiable: "Por eso se exige también hacer sensible un concepto abstracto, es decir, exponer en la intuición el objeto que le corresponde, porque sin esto, el concepto quedaría (como se suele decir) sin sentido, es decir, sin significado" 5 . De acuerdo con las líneas citadas, "Sinn" y "Bedeutung" poseen la misma significación.

Sin embargo, Nolan estima que hay al menos dos pasajes en los que Kant parece establecer una distinción entre los términos mencionados. Uno de esos pasajes pertenece al capítulo del esquematismo y será reproducido más adelante. Allí Kant establece que a las categorías, cuando se hace abstracción de toda condición sensible (sinnlich), les queda aún un significado (Bedeutung) lógico 6 . En el otro pasaje se afirma: “(...) que los conceptos son enteramente imposibles, y no pueden tener significado alguno, si no les es dado un objeto, ya a ellos mismos, ya, por lo menos, a los elementos en que ellos consisten (...)"7. El texto fue enmendado por Kant en su ejemplar impreso. En lugar de “(...) que los conceptos son enteramente imposibles, y no pueden tener significado alguno (...)" debería leerse “(...) que los conceptos no tienen sentido para nosotros, y no pueden tener significado alguno (...)" " Nolan estima que tanto esta enmienda como el pasaje de A 147/B 186 ponen de manifiesto un contraste entre "Sinn" y "Bedeutung". En efecto, parecería que hay aún un problema a resolver en lo que hace al significado de un concepto cuando ya se ha establecido que éste no tiene sentido. De esta afirmación podemos extraer la conclusión de que "Bedeutung" es un concepto más amplio que "Sinn".

Por otra parte, el pasaje de A 240/B 299 citado más arriba nos da un indicio acerca del significado de "Sinn". Allí se afirma que es necesario hacer sensibles (sinnlich $q^{u}$ machen) los conceptos, pues de otra manera ellos quedarían sin sentido (Sinn). Nolan considera, acertadamente a nuestro juicio, que este pasaje pone de manifiesto la afinidad lingüística y conceptual entre sentido (Sinn) y sensible (sinnlich). El sentido de un concepto estaría ligado a la sensibilidad (Sinnlichkeit), y consistiría en tener referencia a un objeto sensible. "Sentido" (Sinn) sería sinónimo de "significado sensible"

\footnotetext{
${ }^{5}$ A $240 /$ B 299.

${ }^{6}$ Cfr. A 147/B 186.

${ }^{7}$ A 139/B 178.

${ }^{8}$ Nachträge LVIII (AA, XXIII, 46).
} 
(sinnliche Bedeutung), "significado empírico" (empirische Bedeutung) y "significado objetivo" (objektive Bedeutung), es decir, una especie del género "significado"9. Nolan establece además que el sentido de un concepto es lo que hace que éste tenga referencia.

A continuación Nolan se ocupa del concepto de logische Bedeutung (significado lógico). Menciona los dos pasajes en los que Kant aborda la cuestión. El primero de ellos es el de A 147/B 186. Lo hemos mencionado más arriba. El segundo pasaje es el de A 248/B 305. Se establece allí que las categorías, sin condiciones formales de la sensibilidad, poseen un significado meramente trascendental (blo $\beta$ transscendentale Bedeutung), aunque carecen de uso trascendental, dado que éste es imposible en sí mismo. Puede afirmarse entonces que las categorías, cuando se hace abstracción de sus esquemas, poseen un significado lógico o trascendental ${ }^{10}$. Por otra parte, la tesis de que las categorías no tienen uso trascendental significa que carece de validez objetiva la aplicación de ellas a las cosas en sí mismas. Señalemos también que en A 244 se da a entender que las categorías puras carecen de significado determinado (bestimmte Bedeutung) ${ }^{11}$.

Presentaremos ahora la reconstrucción efectuada por Z. Loparic de la teoría kantiana de la referencia y el significado. Expondremos sucintamente las tesis generales más relevantes para nuestros fines. Loparic señala que Kant utiliza el término "Beziehung" para mentar la referencia de un concepto a (auf) un objeto ${ }^{12}$. Un concepto que se refiere a un objeto

9 La expresión "sinnliche Bedeutung" (significado sensible) es la única empleada por Nolan. Las dos restantes, en cambio, las introducimos nosotros basándonos en el uso kantiano de las mismas. Cfr. A 197/B 243 y A 499/B 527.

${ }^{10} \mathrm{D}$. Leserre se ha ocupado del problema del concepto de significado empleado por Kant en la "Analítica trascendental" de la Crítica de la razón pura. Además de los niveles objetivo y lógico del significado, Leserre considera que hay un nivel léxico del mismo. El significado, en sentido léxico, no es sino lo que una palabra significa. Cfr. Leserre, D., La comprensión del lenguaje en la Crítica de la razón pura de Kant, Buenos Aires, Centro de Estudios Filosóficos Eugenio Pucciarelli, 2008, pp. 109 ss. ${ }^{11}$ Cfr. Nolan, J. P., op. cit., pp. 113-118. El lector encontrará asimismo en estas páginas una comparación de la concepción kantiana del significado con las reflexiones de $\mathrm{G}$. Frege en torno a esta cuestión.

${ }^{12}$ Cfr. A 286/B 342. 
específico (es decir, no meramente a un objeto en general) tiene significado (Bedeutung) objetivo. Loparic señala que los conceptos que no se refieren a multiplicidades intuitivas específicas, y por tanto tampoco a objetos específicos, conservan un cierto significado, aunque meramente lógico o trascendental. En este caso, Kant califica los conceptos como "vacíos" $(\text { leer })^{13}$. Tales conceptos no se refieren a objetos específicos ni pueden proporcionar conocimiento objetivo, sino que constituyen únicamente la forma discursiva del conocimiento. Con todo, puede afirmarse que poseen un cierto referente. Según Loparic, el referente de los conceptos vacíos debe concebirse como un objeto trascendental, o algo en general $=\mathrm{x}$, es decir, como el pensamiento enteramente indeterminado de un objeto en general ${ }^{14}$. En el objeto trascendental se piensa tan sólo la unidad de la multiplicidad de una intuición en general ${ }^{15}$.

Loparic estima que la relación entre referencia y significado objetivos puede interpretarse de la siguiente manera: un concepto tiene significado objetivo solamente si posee referencia objetiva ${ }^{16}$ (es decir, si puede corresponderle un objeto específico). Esta tesis se opone a la afirmación de Nolan mencionada más arriba. En efecto, para Nolan el sentido (o significado empírico u objetivo) de un concepto es lo que hace que éste tenga referencia. Consideramos que el pasaje de B 302-303 n. demuestra que la interpretación de Loparic es la que más se ajusta a los textos. En esta nota Kant establece que si se suprime toda intuición sensible no se pueden ejemplificar los conceptos, y queda únicamente la posibilidad lógica de los mismos. A continuación se afirma lo siguiente: “(...) no se trata, empero, de esto, sino que se trata de si él se refiere a un objeto, y por tanto, si significa algo"17. Se aprecia aquí cómo el hecho de que un concepto tenga significado

${ }^{13}$ Cfr. A 51/B 75, A 239/B 298.

${ }^{14}$ Cfr. A 104-105, A 109, A 247-248/B 304-305, A 250 y A 253. Señalemos que en otros pasajes el concepto de objeto trascendental hace referencia a la cosa en sí misma. Cfr., por ejemplo, A 379-380 y A 393. Para un análisis de esta ambigüedad, cfr. Allison, H. E., "Kant's Concept of the Transcendental Object", Kant Studien, 59, 2, 1968, pp. 165-186.

${ }^{15}$ Cfr. A 247/B 304.

${ }^{16}$ Cfr. Loparic, Z., op. cit., pp. 171-176.

17 Por su parte, Loparic menciona los pasajes de A 95, A 139/B 178, A 146/B 185, A 238-240/B 298-299. 
es una consecuencia de que posee referencia objetiva. Tal vez sea preciso interpretar "significa" (bedeute) como "significa objetivamente", pues de otra manera el texto entraría en contradicción con la doctrina del significado lógico de las categorías puras. Podemos concluir, entonces, que la referencia objetiva de un concepto es lo que hace que éste tenga sentido o significado objetivo.

Es preciso señalar que Loparic no parece distinguir entre sentido (Sinn) y significado (Bedeutung). En este punto, consideramos que la perspectiva de Nolan es la acertada. En efecto, textos como los citados más arriba muestran que por momentos Kant establece una distinción entre los dos términos.

II.

A continuación procuraremos exponer más detalladamente la teoría del significado lógico de las categorías. En primer lugar nos ocuparemos del pasaje del capítulo del esquematismo en el que Kant trata la relación entre esquemas trascendentales y significado. Posteriormente intentaremos demostrar que, pese a los pasajes que parecen ponerla en entredicho, esta doctrina es parte integrante de la filosofía crítica. Más adelante sostendremos que el significado lógico de las categorías debe concebirse fundamentalmente como indeterminado.

Reproducimos el pasaje más importante del capítulo del esquematismo en lo que hace al problema de la relación entre esquematismo y significado.

Si dejamos de lado una condición restrictiva, parece que amplificáramos el concepto antes limitado; así, las categorías, en su significado puro, sin todas las condiciones de la sensibilidad, deberían tener validez para cosas en general, tales como son, en lugar de lo cual sus esquemas las representan solamente tales como aparecen; y entonces aquellas [deberían aparentementc] tener un significado independiente de todos los esquemas y mucho más extendido. En realidad, es cierto que les queda a los conceptos puros del entendimiento, incluso después de hacer abstracción de toda condición sensible, un significado; pero solamente lógico, [el significado] de mera unidad de las representaciones, a las cuales, empero, no les es dado ningún 
objeto, y por tanto, tampoco significado alguno que pudiera producir un concepto de objeto ${ }^{18}$.

Kant afirma que, si dejamos de lado los esquemas trascendentales, parecería que amplificáramos el ámbito de aplicación de las categorías. Al no estar limitadas por condiciones sensibles, las categorías, aparentemente, nos proporcionarían conocimiento de cosas en general, y no únicamente de objetos dados a nuestra sensibilidad. Sin embargo, esta primera aproximación se muestra incorrecta. En efecto, sin los esquemas trascendentales las categorías no podrían determinar objeto alguno ni, consiguientemente, ser útiles para la consecución del conocimiento ${ }^{19}$. Dicho en otras palabras, las categorías tienen un único uso objetivamente válido, a saber, el empírico, y no pueden aplicarse con validez objetiva a cosas en general o consideradas en sí mismas, es decir, no tienen un uso trascendental ${ }^{20}$.

Ahora bien, en el texto citado se indica que las categorías, cuando se hace abstracción de sus esquemas, conservan un significado (Bedeutung), aunque puramente lógico. Se afirma además que tal significado consiste en la mera unidad de las representaciones y no implica referencia a objetos. Por tanto, si bien las categorías por sí solas no pueden aplicarse a objetos, poseen sin embargo una cierta clase de significado. En síntesis, las categorías no tienen uso objetivo ni significado empírico con independencia de los esquemas trascendentales, aunque no por ello carecen de significado lógico.

Sin embargo, debe señalarse que en $K r V$ se encuentran varios pasajes en los que Kant parece contradecir la doctrina del significado lógico de las categorías puras. Un buen ejemplo es el pasaje de B 302-303 n. citado más

\footnotetext{
${ }_{18}$ A 146-147/B 186. (Tr. modificada).

${ }^{19}$ La expresión "(...) un concepto de objeto (...)", incluida en el pasaje arriba citado, fue corregida por Kant en su ejemplar impreso. Debería leerse de la siguiente manera: "(...) un conocimiento de objeto (...)". AA, XXIII, 46. Por ende, en el texto en cuestión se procura establecer que sin los esquemas trascendentales las categorías no proporcionarían conocimiento objetivo alguno.

${ }^{20}$ Cfr. A 131/B 170, A 139/B 178, A 180/B 223, A 241-242, A 257/B 313, A 259260/B 314-315, A 289/B 345, A 295-296/B 352, A 319/B 376, A 333/B 390, A 650/B 678, A 712/B 740.
} 
arriba. Recordemos que allí se afirma que los conceptos carentes de referencia objetiva no poseen significado ${ }^{21}$. A nuestro juicio, el texto más contundente a este respecto es el de A 136/B 175. Allí se indica que la filosofía trascendental

(...) a la vez debe exponer, mediante características universales pero suficientes, las condiciones bajo las cuales puedan ser dados objetos en concordancia con aquellos conceptos [MA: categorías]; en caso contrario, carecerían de todo contenido, y por tanto, serían meras formas lógicas y no conceptos puros del entendimiento 22 .

En el texto citado se establece que la filosofía trascendental debe exponer las condiciones bajo las cuales pueden ser dados objetos correspondientes a las categorías. Estas condiciones, evidentemente, son los esquemas trascendentales. Ahora bien, a continuación se indica que si no se presentaran tales condiciones las categorías no tendrían ningún contenido, más aún, que serían meras formas lógicas y no conceptos puros del entendimiento. El pasaje no se limitaría a denegar significado lógico a las categorías puras, sino que sugeriría incluso que éstas no tienen ningún contenido y no son siquiera conceptos.

Aparentemente, textos como el citado no dejan al comentarista otra opción que afirmar que Kant realmente no quiso decir lo que dijo ${ }^{23}$. Aun así, procuraremos interpretarlo de modo tal que sea compatible con la doctrina del significado lógico. Kant afirma que sin esquemas

${ }^{21}$ Cfr. también B 148-149.

${ }^{22}$ Tr. modificada. Cfr. también A 147/B 187 y A 348-349.

${ }^{23}$ Esta es la solución que adopta Nolan con respecto a los pasajes que deniegan significado lógico a las categorías puras. "Sus enérgicas declaraciones acerca de la falta de significado de las categorías puras consideradas con independencia de sus esquemas sensibles no han de ser tomadas al pie de la letra. Cuando Kant dice que las categorías puras no tienen significado, no quiere realmente decir eso." Nolan, J. P., op. cit., p. 118. Nolan cita como ejemplos los pasajes de A 145-146/B 185 y A 696/B 724. Este comentarista considera que las imprecisiones de Kant en torno al problema de la semántica de las categorías se deben a que no determinó suficientemente su concepto de significado lógico. 
trascendentales las categorías no tendrían contenido. Ahora bien, en $\mathrm{KrV}$ el término "contenido" equivale a menudo a "referencia a intuiciones humanas espacio-temporales" 24 . Por tanto, es posible interpretar que Kant se está limitando a establecer que las categorías puras no se refieren inmediatamente a intuiciones humanas. Dicho en otras palabras, las categorías puras son conceptos vacíos, es decir, a ellas no les corresponden inmediatamente objetos de nuestra intuición. Tal afirmación no implica un rechazo de la tesis del significado lógico de las categorías puras.

Más dificultades presenta la tesis de que sin esquemas trascendentales las categorías serían meras formas lógicas y no conceptos puros del entendimiento. Aunque, posiblemente, el texto no pretenda afirmar exactamente eso. El texto señala que la filosofía trascendental debe exponer (muß ... darlegen) las condiciones que permiten el empleo objetivo de las categorías, y que de lo contrario las categorías serían únicamente formas lógicas y no conceptos. Ahora bien, la afirmación puede entenderse en el sentido de que Kant está contemplando la posibilidad de que las categorías no sean susceptibles de ser esquematizadas. No estarían en cuestión las categorías puras, es decir, consideradas haciendo abstracción de los esquemas trascendentales, sino unas hipotéticas categorías no esquematizables. El pasaje establecería únicamente que, si no fuera posible producir esquemas correspondientes a las categorías, ellas no serían conceptos puros del entendimiento.

No es nuestra intención afirmar que la interpretación ofrecida del pasaje mencionado resuelve de manera inequívoca todos los problemas que presenta. Y aun si fuera así, en A 696/B 724 Kant afirma que fuera del campo de la experiencia posible las categorías son meros títulos de conceptos $^{25}$. Además, queda en pie la objeción de que en otros textos Kant deniega a las categorías puras todo tipo de significado.

${ }^{24}$ Acerca de este punto, cfr. Caimi, M., "Gedanken ohne Inhalt sind leer", Kant Studien, 96, 2, 2005, pp. 135-146; y Young, M., "Synthesis and the Content of Pure Concepts in Kant's First Critique", Journal of the History of Philosophy, 32, 3, 1994, pp. 331-357.

${ }^{25}$ Cfr. también A 679/B 707. El lector encontrará una interpretación diferente de los pasajes mencionados en un trabajo de R. E. Aquila. Aquila sostiene que Kant 
Sin embargo, consideramos que la doctrina del significado lógico de las categorías puras es una genuina enseñanza kantiana. En efecto, esta doctrina está en armonía con otras tesis importantes de la filosofía crítica. Está en concordancia, en primer lugar, con la independencia del entendimiento con respecto a la sensibilidad ${ }^{26}$. En segundo lugar, la doctrina que nos ocupa parece ser una consecuencia de la distinción efectuada en el $\S$ 24 de $\mathrm{KrV}$ entre síntesis intelectual y síntesis figurada. Si las categorías no conservasen un significado con independencia de sus esquemas, no tendría

nunca no tuvo gran claridad acerca de la relación entre formas del juicio y conceptos categoriales. La confusión tendría su origen en el hecho de que las formas del juicio tienen ya un cierto çontenido semántico, a saber, los modos en que un concepto se encuentra presente en la conciencia. Por este motivo Kant habría vacilado en cuanto a considerarlas como conceptos. Cuando Kant entendía que las formas del juicio eran conceptos, las llamaba categorías puras. Esta circunstancia explicaría que Kant negara, en algunos pasajes, la existencia de categorías no esquematizadas y afirmara, en otros, que sin esquemas trascendentales tenemos aún categorías puras. Según Aquila, el esquematismo trascendental sería en última instancia el proceso mediante el cual las categorías puras o formas del juicio se transforman en categorías fenoménicas o esquematizadas. Cfr. Aquila, R. E., "Kategorien, Schematismus und Urteilsformen", Ratio, 18, 1, 1976, pp. 29-45. T. K. Seung, por su parte, considera que Kant tenía dos concepciones de las categorías, una genérica y otra lógica. Según la primera, las categorías son conceptos de un objeto en general, es decir, los géneros últimos. Según la segunda, las categorías son formas del juicio. La primera concepción de las categorías puede entenderse como su función real, mientras que la segunda, como su función lógica. Parte de las dificultades hermenéuticas que presenta el Capítulo del esquematismo se deberían a que Kant, en ese texto, emplea alternativamente sus dos concepciones de las categorías. Cfr. Seung, T. K., "Kant's conception of the categories", Review of Metaphysics, 43, 1, 1989, pp. 107-132. Nuestra objeción a los dos artículos mencionados es la misma. Consiste en que no toman en cuenta la caracterización de la relación entre categoría pura y función lógica formulada en B 128. Allí se afirma que las categorías son conceptos de un objeto en general, por intermedio de los cuales la intuición de éste se considera como determinada con respecto a una de las funciones lógicas del juzgar. Las categorías puras, por tanto, si bien tienen un contenido semántico no fenoménico que depende de las funciones lógicas, no son idénticas a ellas ni a las formas del juicio en las cuales tales funciones se expresan. Cfr. infra.

${ }^{26}$ Cfr. A 254/B 309 y A 287/B 343. 
sentido hablar de una síntesis meramente intelectual. En tercer lugar, el significado lógico de las categorías garantiza que el pensar tenga mayor extensión que el conocer. Pensar no es lo mismo que conocer. Sólo es posible conocer un objeto si a los conceptos les corresponden intuiciones. En cambio, para pensar se requieren únicamente conceptos. Por este motivo podemos pensar cualquier cosa, siempre y cuando nuestro concepto no encierre contradicción. Ahora bien, a fin de que con las categorías sea posible, no tanto conocer, sino solamente pensar, es preciso que ellas conserven un significado cuando se las considera con independencia de sus esquemas ${ }^{27}$. En cuarto lugar, sostenemos que la doctrina mencionada es parte integrante de la doctrina del esquematismo trascendental. En efecto, ¿qué es lo que haría que a una categoría pura le corresponda un esquema determinado, y no otro, si ella no tuviera significado alguno? Suele afirmarse que los esquemas trascendentales son interpretaciones temporales de las categorías. $\mathrm{Y}$, ¿qué es lo que se expresaría en términos de tiempo si las categorías puras no tuviesen significado? ${ }^{28}$

En conclusión, consideramos que el significado lógico de una categoría pura se deriva de lo pensado en ella, a saber, de aquello que, en términos de Logik Jäsche, podría denominarse el contenido de un concepto ${ }^{29}$. Ciertamente no es posible conocer un objeto mediante una categoría pura, aunque es innegable que algo es pensado en ella. Tal vez los pasajes en los

27 Para la distinción entre pensar y conocer, cfr. B XXVI n., B XXVIII, B 146, A 154-156/ B 194-195 ss. Señalemos que D. Dahlstrom ha interpretado la doctrina del esquematismo en términos de la diferencia entre pensar y conocer. Para que el pensamiento representado por un concepto, en tanto género, se transforme en conocimiento, en cuanto especie, sería necesario añadirle la diferencia específica en la que consiste su esquema. Notemos que la interpretación de Dahlstrom tiene en cuenta ante todo el esquematismo de los conceptos empíricos. Cfr. Dahlstrom, D., "Thinking, Knowing, and Schematism", en Funke, Gerhard (ed.), Akten des 5. Internationalen Kant-Kongresses, I, 1, Bonn, Bouvier Verlag Herbert Grundmann, 1981, pp. 209-220, aquí pp. 218-220.

${ }_{28}$ I a tesis del significado lógico de las categorías puras, cuanto menos de la categoría de la causalidad, es indispensable asimismo para la filosofía práctica kantiana. En efecto, en este ámbito es legítimo un uso, si bien no cognitivo, de las categorías puras. Cfr. KpV, "Vorrede" (AA, V, 5-6, 65).

${ }^{29}$ Cfr. Logik Jäsche, $\int 7$ (A A, IX, 95). 
que Kant niega que las categorías puras posean significado puedan ser interpretados a la luz de su doctrina de los conceptos vacíos. En efecto, Kant califica los conceptos que no se refieren a intuiciones humanas como vacíos y sin contenido ${ }^{30}$. Esta circunstancia se debe a que, pese a que es posible que en tales conceptos algo sea pensado, ellos no son útiles para la tarea del conocimiento. Podría interpretarse entonces que Kant, cuando afirma que las categorías puras carecen de significado, quiere dar a entender únicamente que carecen de significado objetivo en virtud de que no se refieren a intuiciones humanas, y que por tanto carecen de significado para la prosecución del conocimiento.

\section{III.}

Ahora bien, aunque parezca indiscutible que la doctrina del significado lógico es parte integrante de la filosofía crítica $^{31}$, no es tarea

${ }^{30}$ Cfr. Caimi, M., op. cit.

${ }^{31}$ R. P. Wolff considera en cambio que la doctrina del esquematismo en general, y la distinción entre categoría pura y esquema trascendental en particular, fueron elaboradas únicamente en virtud de las siguientes motivaciones: 1) la preocupación por mantener la disposición "arquitectónica" del sistema, 2) el interés ćtico y teológico de Kant, y 3) la concepción de las categorías como conceptos de aplicación problemática a las cosas en sí mismas, concepción que en última instancia se deriva del período pre-crítico. La verdadera doctrina de la Analítica de $\mathrm{KrV}$ consistiría en que las categorías son modos de la conciencia del tiempo, y que por tanto se extienden únicamente hasta donde lo permite la sensibilidad. Más allá del ámbito de la sensibilidad y la temporalidad, las categorías no conservarían significado alguno (become meaningless). Cfr. Wolff, R. P., Kant's Theory of Mental Activity, Gloucester, Peter Smith, 1973, pp. 214-218. Las tesis de que el capítulo del esquematismo contiene argumentos "artificiales" (artificial) y de que su inclusión en $\mathrm{KrV}$ fue motivada por preocupaciones "arquitectónicas" (architectonic) habían sido presentadas por N. Kemp Smith. Cfr. Kemp Smith, N., A Commentary to Kant's "Critique of Pure Reason", New York, Humanity Books, 1999 (1923), pp. 334-336. En la p. XXII de su obra Kemp Smith explica que por "arquitectónica" entiende el plan general de $\mathrm{Kr} V$ en tanto basado en principios declaradamente lógicos. Tal arquitectónica daría a $\mathrm{KrV}$ la apariencia de un orden sistemático, incluso cuando ese orden está ausente. Señalemos que, por su partc, Kant caracteriza la arquitectónica como el arte de los sistemas. Cfr. A 832/B 860. 
sencilla precisar en qué consiste el significado lógico de las distintas categorías puras. Posiblemente el pasaje más importante para este problema sea el de A 244-245. Allí se afirma que las categorías puras carecen de significado determinado. Sin esquemas, no es posible discernir qué objetos pueden someterse a las categorías. Las categorías puras, indica Kant, no son conceptos mediante los cuales un objeto sea conocido ni distinguido de otros, sino únicamente maneras de pensar un objeto de intuiciones en general y de darle un significado según una función lógica del entendimiento.

Es preciso considerar la caracterización de las categorías presentada en B 128. Según se dice allí, las categorías son conceptos de un objeto en general, mediante los cuales la intuición de éste se considera como determinada con respecto a una de las funciones lógicas del juzgar. La comprensión de esta tesis presupone una breve explicación del concepto kantiano de función. Brindaremos esa explicación a continuación.

Una primera aproximación al concepto kantiano de función nos revela que éste es empleado por Kant en un sentido fisiológico. El entendimiento tiene una función en el mismo sentido en que un órgano tiene una tarea que le corresponde desempeñar. Así como la función del ojo es ver, la función del entendimiento es juzgar. Sin embargo, hay que destacar que Kant no se limita a emplear el término "función" en un sentido fisiológico, sino que ofrece una definición del concepto de función. Una función es la unidad de una acción. ${ }^{32}$ A diferencia de las acciones, las unidades de acción no son eventos localizables en el tiempo. Una misma unidad de acción puede ser ejercida en diversas acciones concretas. ${ }^{33}$ Además, una unidad de acción puede ser un complejo de diversas unidades de acción. Ilustraremos estas tesis con el ejemplo fisiológico del ojo. El ver, como función del ojo humano, puede ser comprendido como la unidad idéntica de diversas acciones concretas. Tal unidad de acción encierra en sí

32 Cfr. A 68/B 93.

33 M. Caimi afirma que una función es una acción considerada no tanto como acontecimiento único, sino más bien en su especificidad. Una acción considerada en su especificidad no es sino la "unidad de la acción", que en tanto una y la misma acontece una y otra vez. Cfr. Caimi, M., "Einige Bemerkungen über die Metaphysische Deduktion in der Kritik der reinen Vernunft', Kant Studien, 91, 3, 2000, pp. 257-292, aquí p. 266, n. 36. 
misma, como momentos, funciones subordinadas tales como el experimentar colores y el percibir figuras ${ }^{34}$.

De acuerdo con la explicación precedente, la función del entendimiento es juzgar. Esta función se ejerce en diversas acciones concretas y encierra funciones subordinadas. Según Kant, tales funciones subordinadas se dividen en cuatro clases, a saber, las de la cantidad, la cualidad, la relación y la modalidad. Cada una de estas clases, a su vez, contiene tres elementos. Por lo tanto, hay doce funciones lógicas del entendimiento en los juicios o funciones del juzgar.

Cabe destacar que el ejercicio de una función del entendimiento confiere a un juicio una forma determinada. Los juicios, como consecuencia del ejercicio de una determinada función lógica del entendimiento humano, reciben la forma correspondiente a esa función. Por ejemplo, la función del entendimiento ejercida en los juicios categóricos confiere a estos juicios la forma lógica consistente en la relación de acuerdo u oposición entre un concepto sujeto y un concepto predicado, a saber, "S es P" o "S no es P". En síntesis, el ejercicio de una función del entendimiento produce la forma del juicio correspondiente, y esta última expresa la primera.

Kant considera que cada función del juzgar origina, además de una forma del juicio, un concepto puro del entendimiento. Las categorías y las formas del juicio surgen por parejas de las mismas funciones del juzgar ${ }^{35}$. Más precisamente, según Kant, la misma función que da unidad a diversas representaciones en un juicio, da unidad a la síntesis de diversas representaciones en una intuición. Puede afirmarse, entonces, que las funciones del entendimiento producen dos tipos diferentes de unidad. En primer lugar, una función del entendimiento unifica conceptos en juicios de una determinada forma lógica. Por ejemplo, la función del entendimiento ejercida en los juicios categóricos puede unificar los conceptos "hombre" y "mortal" en el juicio "todos los hombres son mortales". En virtud del ejercicio de la función mencionada, el juicio citado obtiene la forma de los

${ }^{34}$ Nuestra explicación del concepto de función sigue de cerca la de M. Wolff. Cfr. Wolff, M., Die Vollständigkeit der kantischen Urteilstafel. Mit einem Essay ïber Freges Begriffsschrift, Frankfurt am Main, Vittorio Klostermann, 1995, pp. 20-22.

${ }^{35}$ Cfr. A 79/B 104-105. 
juicios categóricos. En segundo lugar, una función del entendimiento da unidad a la síntesis de diversas representaciones en una intuición. "Dar unidad" a la acción de síntesis significa determinar como necesaria la unión de los elementos que la síntesis ha de reunir. Las categorías son precisamente los conceptos que representan la mencionada unidad de la síntesis. Dicho en otras palabras, las categorías son conceptos que representan la unión o pertenencia recíproca necesarias de los elementos que debe reunir la acción de síntesis. Por ejemplo, la categoría de inherencia y subsistencia representa la unidad necesaria de los elementos de la multiplicidad intuitiva en la medida en que son accidentes de una substancia.

Retomemos ahora la caracterización de las categorías de B 128: las categorías son conceptos de un objeto en general, mediante los cuales la intuición de éste se considera como determinada con respecto a una de las funciones lógicas del juzgar. Tomaremos como ejemplo la función lógica ejercida en los juicios hipotéticos. En tales juicios se da una conexión entre el fundamento y la consecuencia. Un ejemplo de juicio hipotético es: "si los cuerpos son compuestos, entonces son divisibles". La forma de ese juicio hipotético viene dada por el tipo de conexión que se presenta entre el fundamento y la consecuencia. El fundamento es: "si los cuerpos son compuestos", y la consecuencia es: "entonces son divisibles". La forma del juicio hipotético no es sino la conexión: "si ... entonces ...".

Mediante la categoría pura de causalidad y dependencia se piensa la función lógica ejercida en los juicios hipotéticos en tanto determina una intuición en general. En síntesis, el significado lógico de esta categoría pura consiste en la referencia determinante de la unidad lógica del fundamento y la consecuencia a objetos de una intuición en general. Dicho en otras palabras, la categoría pura de causalidad y dependencia significa objetos de una intuición en general en tanto determinados por la relación lógica "si ... entonces".

Es evidente que tal significado, como afirma Kant, permanece indeterminado. No tenemos un concepto acerca de un modo de intuición diferente del nuestro, a saber, el espacio-temporal ${ }^{36}$. Por otra parte, la intuición en general es una intuición cuyo modo de ser permanece no

${ }^{36}$ Cfr. B 139 y A 230/B 283. 
especificado. Por tanto, sin la referencia a intuiciones humanas proporcionada por los esquemas trascendentales, las categorías no poseerían significado determinado alguno.

Retomemos el ejemplo de la categoría de causalidad y dependencia. En A 243/B 301 Kant indica que en esta categoría pura se piensa meramente algo a partir de lo cual se puede inferir la existencia de algo diferente. Ciertamente, el significado lógico de la categoría de causalidad y dependencia es enteramente indeterminado. Por un lado, "algo" (etwas) no hace referencia a un objeto de nuestra intuición espacio-temporal, sino a un objeto en general. Por otro lado, no sabemos cómo debe interpretarse espacio-temporalmente el "inferir" (schließen) la existencia de algo a partir de la existencia de algo diferente. En síntesis, sabemos únicamente que objetos de intuiciones en general tienen que estar conectados mediante un enlace intuitivo análogo a la relación de fundamentación lógica ("si ... entonces"). Ahora bien, en la medida en que el modo de ser de estas intuiciones no es especificado, el significado lógico de la categoría pura de causalidad y dependencia permanece indeterminado. Como afirma Kant en el pasaje de A 244-245 mencionado más arriba, las categorías puras no tienen significado determinado y tan sólo conceden significado a un objeto de una intuición en general con respecto a una función lógica del entendimiento ${ }^{37}$.

Hemos sostenido, en primer lugar, que la doctrina del significado lógico de las categorías puras es parte integrante de la filosofía crítica kantiana. En segundo lugar, hemos mostrado que ese significado lógico debe concebirse ante todo como indeterminado. En efecto, en este significado se hace abstracción de la referencia de las categorías a intuiciones determinadas espacio-temporales que es posibilitada por los esquemas trascendentales.

Recibido: 07/2010; aceptado: 09/2010.

${ }^{37}$ Señalemos que el significado empírico de la categoría de causalidad y dependencia es determinado. Gracias al esquema trascendental de esta categoría, la relación de fundamentación lógica pensada en la categoría pura es expresada temporalmente en la sucesión universal y necesaria de los fenómenos. Los esquemas trascendentales no son sino la expresión temporal del significado lógico de las categorías puras. 